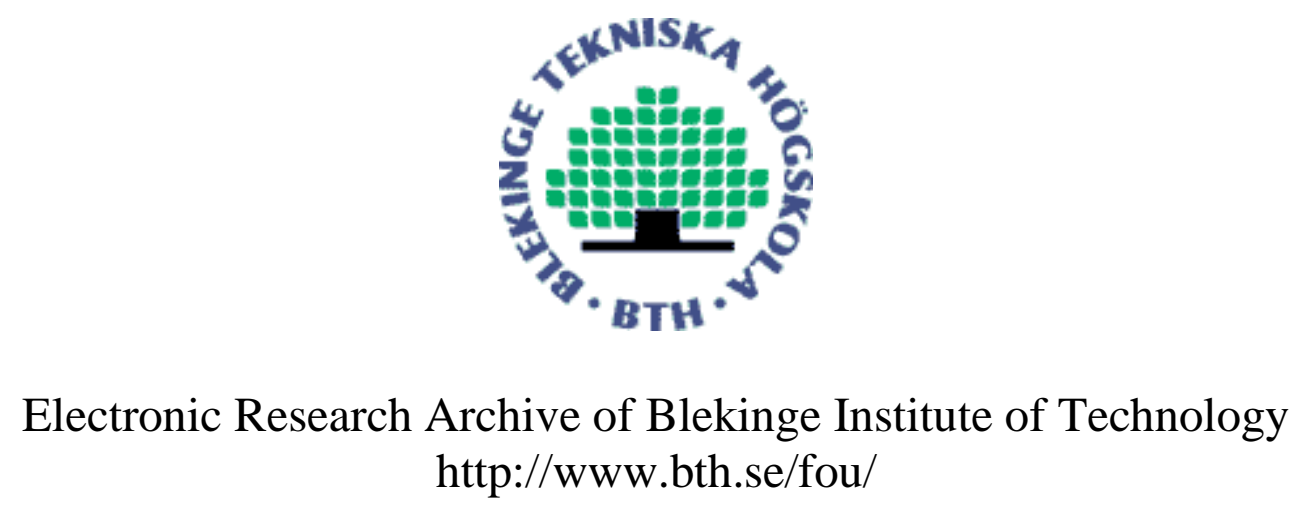

This is an author produced version of a conference paper. The paper has been peer-reviewed but may not include the final publisher proof-corrections or pagination of the proceedings.

Citation for the published Conference paper:

Title:

Comparing a Hybrid Testing Process with Scripted and Exploratory Testing: An Experimental Study with Practitioners

Author:

Syed Muhammad Ali Shah, Usman Sattar Alvi, Cigdem Gencel, Kai Petersen

Conference Name:

Agile Processes in Software Engineering and Extreme Programming - 15th International Conference (XP)

Conference Year:

2014

Conference Location:

Rome

Access to the published version may require subscription.

Published with permission from:

Springer International Publishing 


\title{
Comparing a Hybrid Testing Process with Scripted and Exploratory Testing: An Experimental Study with Practitioners
}

\author{
Syed Muhammad Ali Shah, Usman Sattar Alvi, Cigdem Gencel, Kai Petersen \\ Politecnico di Torino, 2410129 Torino, Italy \\ Seamless AB, Stockholm, Sweden \\ Free University of Bolzano-Bozen, 39100 Bolzano (Bozen), Italy \\ Blekinge Institute of Technology, Karlskrona, Sweden \\ syed.shah@polito.it, usman.alvi@seamless.se, cigdem.gencel@unibz.it, \\ kai.petersen@bth.se
}

\begin{abstract}
This study presents an experimental study comparing the testing quality of a Hybrid Testing (HT) process with the commonly used approaches in industry: Scripted Testing (ST) and Exploratory Testing (ET). The study was conducted in an international IT service company in Sweden with the involvement of six experienced testers. Two measures were used for comparison: 1) defect detection effectiveness (DDE) and 2) functionality coverage (FC). The results indicated that HT performed better in terms of DDE than ST and worse than ET. In terms of FC, HT performed better than ET, while no significant differences were observed between the HT and ST. Furthermore, HT performed best for experienced testers, but worse with less experienced testers.
\end{abstract}

Key words: Exploratory Testing, Scripted Testing, Hybrid Testing, Experiment, Industrial

\section{Introduction}

The common testing process in industry is scripted testing (ST) (also referred to as prescriptive or test case based testing [14]). ST is a plan-driven testing process where test cases are designed prior to test execution in order to structure and to guide the testing tasks. This approach provides several benefits such as test awareness, test coverage, repeatability, and tracking [1],[17].

However, some recent studies indicate that the use of rigorous and welldocumented ST is not very common [2],[3],[18]. One reason stated by Itkonen [15] was that documenting every scenario in a test case being very time consuming and hence testers requiring more time in writing test cases as compared to actually executing them. Furthermore, Andersson and Runeson [1] mentioned that testers do not often rely on the test cases while actually executing them.

Furthermore, Itkonen [15] mentioned that the actual effectiveness and importance of these pre-designed test cases in terms of defect detection efficiency is also unknown. Agruss et al. [1] and Andersson et al. [3] claimed that if all 
the pre designed test cases pass in the first execution, chances of finding any new bugs by executing the same test set again are nominal. Kaner [21] described another limitation of using predesigned test cases as the need to redesign the test cases for every new version of the software.

Exploratory testing (ET) is another testing approach, which has also become popular particularly in the agile world [11],[19]. In ET, tests are not planned and defined in advance, but are dynamically designed, executed, and modified [7]. As defined by [5] "Exploratory testing is simultaneous learning, test design, and test execution". Therefore, ET embraces similar values as agile development and combines learning, test design, and test execution into one test approach [11]. Following this approach, testers can freely explore an application by utilizing human intuition and experience [4],[24]. As it is not explicit how testers make this exploration, ET is often referred to as 'ad hoc' testing [1]. Recently, the term 'exploratory' was introduced by a group of testing experts instead of 'ad hoc' [5] to avoid wrong perception of this approach.

A few studies such as [9],[16],[17] mentioned that ET makes better use of testers creativity and skills to discover the bugs that ST may not uncover because of its mechanical nature. On the other hand, some shortcomings of the ET approach have been also reported such as; the difficulty in assessing whether all features are tested, unavailability of oracles, and invisibility of the quality of testing [16],[17],[18]. Furthermore, Agruss et al. [1] stated that ET is not suitable while performing acceptance testing.

In our previous study [23], we made a comprehensive review of the strengths and weaknesses of ST and ET by performing a systematic literature review and conducting interviews with testing experts in industry. One of the significant findings of our study was that the performance of ST and ET with respect to testing quality depend on a number of conditions. For example, quality of testing when using ST depends on the test case design, which depends on the skills, experience, and domain knowledge of the test designers as well as the previous documents from which the product requirements are inherited. On the other hand, the quality of the testing when using ET depends on skills, experience, and domain knowledge of the testers who execute the tests.

Therefore, a number of researchers and practitioners [1],[9],[17] highlighted that organisations can benefit more if they use ET in addition to ST, as they are in fact complementary approaches. In general, there is a general interest in industry for a hybrid testing (HT) approach unifying the two approaches, which is, for example, visible in lively discussions in industry oriented blogs. Therefore, in our previous study [23], we designed a hybrid testing (HT) process incorporating the different aspects of ST and ET to mitigate the weaknesses of one by leveraging on the benefits of the other.

In this study, we empirically evaluated the test design and test execution phases of the HT process through an experimental study in a software company. Our research question was formulated as "How does a hybrid testing process affect testing quality as compared to ST and ET?" 
This paper is organised as follows. In Section 2, we provide a short description of the HT process that this study is based on. In Section 3, we present our research methodology. The results are presented and discussed in Section 4. The conclusions are given in Section 5.

\section{Background}

This section provides background information on the HT process [23] (Figure 1), which we evaluated in the experimental study in terms of testing quality. Here, we do not discuss the whole process defined, but only provide background on test design and implementation and test execution sub-processes. The other subprocesses were not in the scope of the experimental study. A detailed account of the overall process and the differences between them is given in [23], which forms the foundation for this work.

Test Design $\mathcal{E}$ Implementation: In HT test design, Requirement Based Test Cases (RBTC) [26] and test missions are introduced to enable both high functionality coverage and defect detection effectiveness in addition to cost effectiveness through reducing the test bed size. RBTC specify those test cases that are defined only from the requirements specification. The "test mission" is a concrete instruction for testing and the problem being looked for.

Test Execution: The test execution part is highly flexible. Both RBTCs and the test missions are executed, which were designed in test design phase. First, a tester has given the freedom to freely explore the application in order to learn and get knowledge about it. After that, RBTCs and then the test missions are executed and the execution artifacts are recorded. A session is an uninterrupted block of test time slot assigned to a specific test mission in which test mission has to be executed.

\section{Method}

We evaluated the test design and test execution phases of the HT process in terms of testing quality (defect detection effectiveness and functionality coverage) through an experimental study. The aim of the evaluation was to test the introduction of HT under a realistic scenario from an industrial standpoint where practitioners first use ST and then migrate to ET and thereafter to HT where different versions of the same software are tested, each version having different defects. Our main research question (RQ) was as follows: RQ: How does a hybrid process affect the testing quality (DDE, FC) in comparison to ST and ET?

Overall, by using the template of Goal Question Metric paradigm [6], the intend of the experimental study can be summarized as:

- Analyze the introduction of ET and HT in an organization originally using $S T$ for the purpose of evaluation and proposition generation 


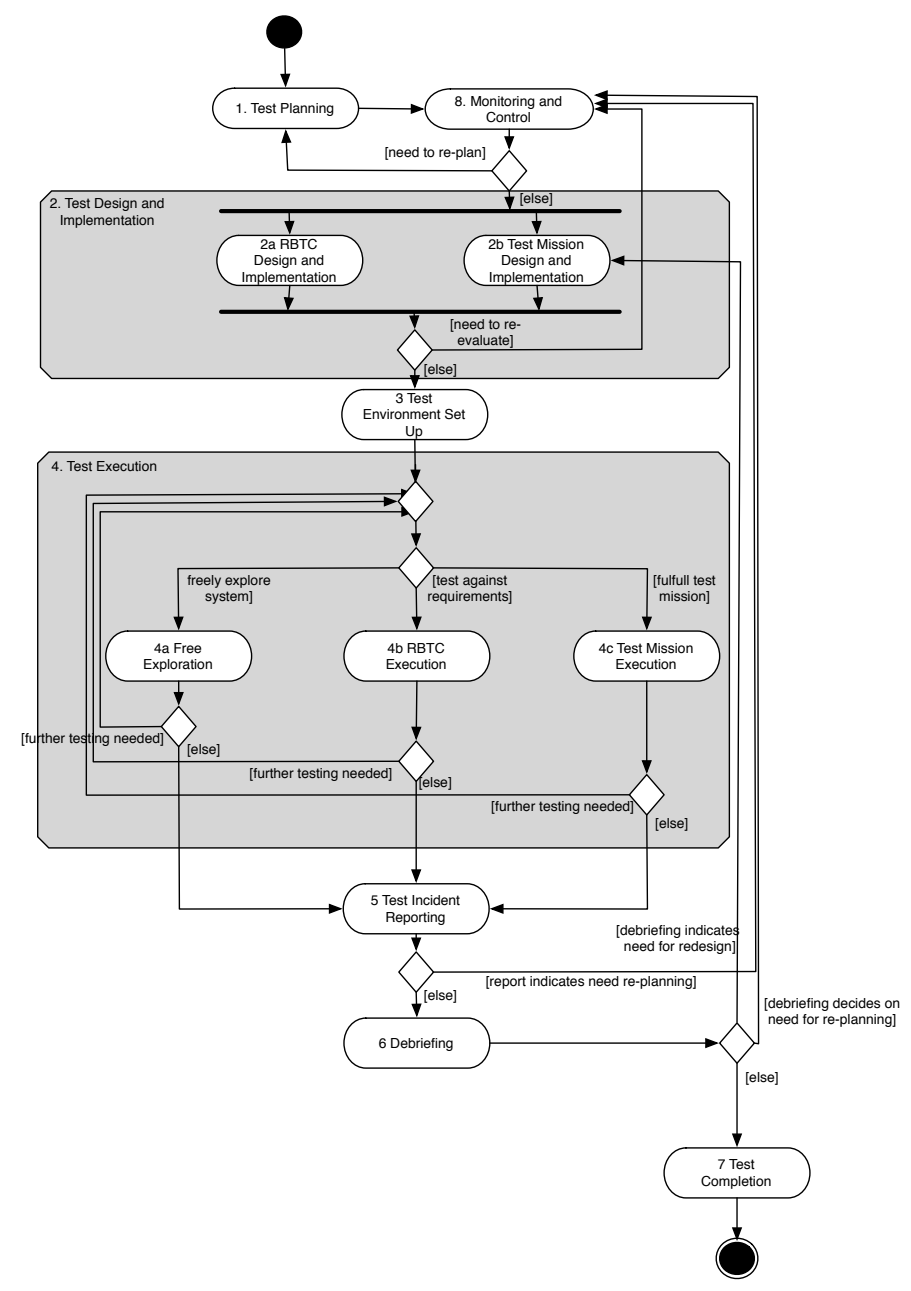

Fig. 1. HT Process

- With respect to defect detection effectiveness (DDE) and functionality coverage $(F C)$

- From the point of view of the researcher

- In the context of experienced industry practitioners (at least 3 years of experience) using the testing approaches from a black box testing perspective on different versions of a calculator software with different bugs seeded.

With respect to testing techniques this means that no white-box approaches were applied, such as covering statements, branches, or decisions. From a blackbox perspectives the practitioners freely explored the application using ET, no techniques were prescribed for that. The same applies to HT where test mission 
statements were used. Test coverage was hence measured in terms of functionality coverage.

The null and alternative hypotheses for the experiment were as follows:

- Detect Detection Effectiveness (DDE)

- $H 1_{0}$ : There is no significant difference between HT, ST and ET

- $H 1_{1}$ : DDE by $\mathrm{HT}\left(\mathrm{DDE}_{H T}\right)$ is higher than by ST $\left(\mathrm{DDE}_{S T}\right)$

- $H 1_{2}$ : DDE by $\mathrm{HT}\left(\mathrm{DDE}_{H T}\right)$ is higher than by $\mathrm{ET}\left(\mathrm{DDE}_{E T}\right)$

- Functionality Coverage (FC)

- $H 2_{0}$ : There is no significant difference between HT, ST and ET

- $H 2_{1}$ : $\mathrm{FC}$ by $\mathrm{HT}\left(\mathrm{FC}_{H T}\right)$ is higher than by $\mathrm{ST}\left(\mathrm{FC}_{S T}\right)$

- $\mathrm{H}_{2}$ : $\mathrm{FC}$ by $\mathrm{HT}\left(\mathrm{FC}_{H T}\right)$ is higher than by $\mathrm{ET}\left(\mathrm{FC}_{E T}\right)$

Experimental Planning and Design: This experimental study was conducted at Logica AB in Sweden (Logica is currently owned by CGI). Logica AB was an international IT service company that creates value by integrating people, business and IT. It had around 39,000 employees, 5,200 of who work in Sweden. The company provided business-consulting, systems integration, development, testing and outsourcing services to all around the world.

We designed the experiment as a "One factor with more than two treatments" [27]. The factor here is the testing approach used with three treatments: ST, ET and HT. The main differences between the three treatments are described in Table 1.

Table 1. Treatment characterization and examples

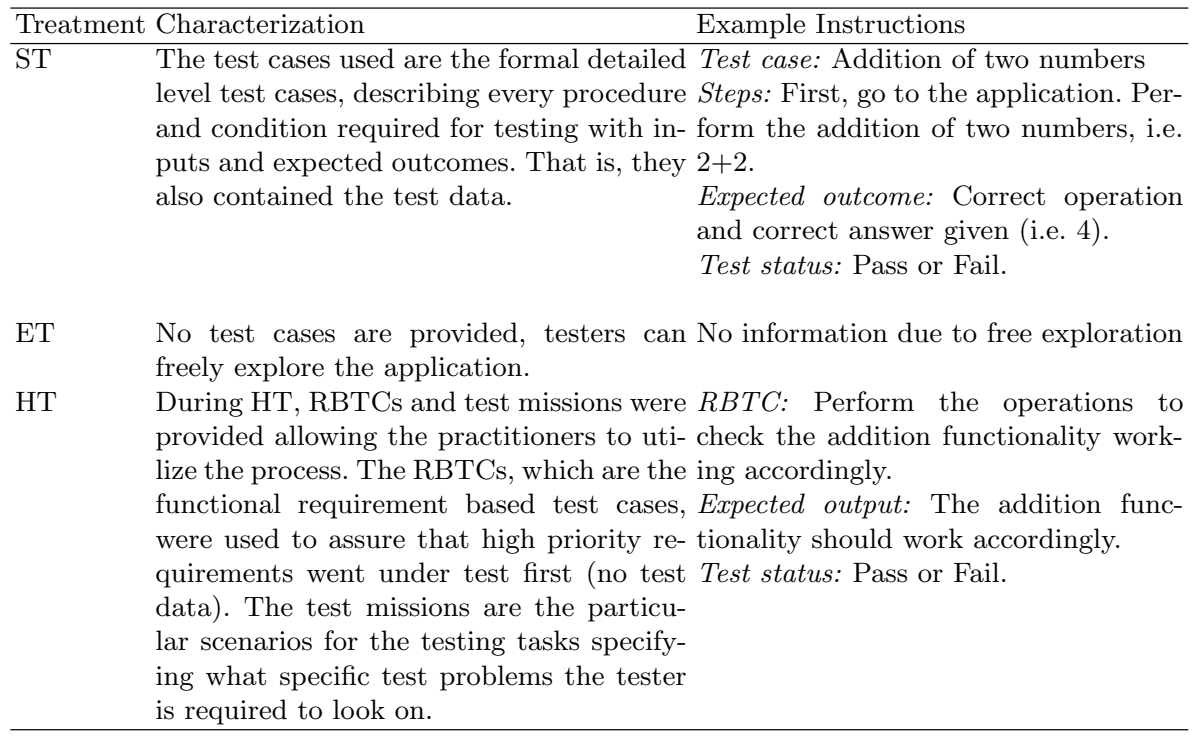

Application: The case application was a calculator application with 11 functions developed by the first two authors of this paper. One application with three versions was developed with the same functionality, but was seeded with different defects for each of the experiment sessions. That is, if in one version (e.g. 
seed 1) division is working without defects, this might not be the case in another version (e.g. seed 2 or seed 3). In total, 25 defects were seeded into each version of the application. The defects were not the same per application, but were of similar types. That is, the testers would not benefit by figuring out a defect once and then could easily discover the same defect with a different testing process. A colleague not involved in the experiment to avoid biasing the results did the seeding of defects. The only information provided to that person was that different types of defects should be seeded, e.g. cosmetic, quality requirements such as response time, functional defects, etc.

The application was tested using ST, ET, and HT, consecutively. This application was chosen to ensure that the practitioners were familiar with the application domain so that there would not be learning effect when they tested the application first with ST, secondly with ET, and finally with HT. In other words, they were as familiar with the application in the beginning as in the end.

Furthermore, for practical reasons, the choice of a calculator application means that the testers would test a small set of functions of an application they are familiar with and have domain knowledge about within a limited amount of time available in the company. However, the alternative, i.e. choosing a version of the systems developed at the company, would not have been feasible due to the lack of experimental control, e.g. with respect to defect population and other factors that would influence/bias the testers in their testing. Therefore, we suggest using the results of this study as an input for proposition generation when conducting future studies (for example in the form of a case study) to learn more about the longitudinal effects of introducing HT into practice.

Test cases: For ST implementation, the designed test cases were provided to the testers. The test cases were written before seeding the defects. That way we avoided writing test cases biased towards the seeded defects. The test cases were documented in the form of test scenarios stating pre-conditions, scenario steps, and post conditions. An example for a scenario step is to conduct a calculation with mixed operations (e.g. multiplication and addition). According to our prestudy and without time limitation the test cases, provided to the subjects for ST and HT, would have identified most of the defects in each seed.

Subjects: The subjects of the experiment were selected from the same company. Giving the potential experiment subjects a questionnaire; we identified 6 subjects considering their experience: T1 (5 years); T2 \& T4 (more than 5 years); T3 \& T5 (test managers with more than 10 years) and T6 (more than 3 years). The same subjects having similar backgrounds participated in the experiment for each treatment to reduce measurement error due to subject heterogeneity, and thus to increase statistical power when sample size is small [13].

Observed Outcome Metrics: In order to be able to compare the testing quality of HT to ST and ET, we chose to use two metrics: (1) 'defect detection effectiveness (DDE)', and (2) 'functionality coverage (FC)'. Both metrics are in ratio scale and, therefore could be used in further statistical tests. Here, DDE corresponds to total number of detected defects out of all seeded defects. We 
talk about defect detection effectiveness instead of efficiency as time has not been considered as a factor in comparing the outcome.

FC corresponds to total number of incorrectly implemented or missing functions detected out of all functions implemented in the software. However for the experiment, all functions of the application were seeded with some defects. Therefore, in this case, FC is the number of detected functions with defects out of the total defective functions. For example, if 6 functionalities are identified to have defects out of 11 functions implemented, this means that the testers achieved $55 \%$ functionality coverage. We should note that, we did not need to normalize these values against the number of total seeded defects and total number of functions, as these were the same for all versions of the Calculator application. Therefore, for DDE, we only report the total number of defects detected whereas for FC, the total number of incorrectly implemented or missing functions detected.

Experiment Execution: Before the experiment date, we gave an initial presentation about the experiment and general guidelines for how to perform the tests using each testing approach. All three versions of the test application were deployed to the laptops of the subjects as well. As the subjects had no access to the source code of the application, this ensured that testers conducted a black-box test of the application.

The experiment was held in two sessions (training and test execution) and totally took two hours. During the first session, we gave detailed instructions for each test approach. Then we provided the subjects with templates for bug investigation report, session sheet and RBTC forms, which were designed to collect experiment related data such as bugs, types of bugs and functionality tested, etc. The first session lasted for 15 minutes and extra 15 minutes were given for additional questions in order to clarify any ambiguities. The subjects were requested not to communicate during the experiment with other testers and concentrate only on the experiment.

The second session was the actual experiment execution session. Maximum 30 minutes was allocated to execute tests using each testing approach. In this session, first, Calculator application with seed 1 was tested using ST. The predesigned test cases were provided to the subjects. It was explicitly mentioned to strictly follow the test steps in the test cases and in case they felt a need to divert from the test steps they were asked to state so in the bug report with the reasons and any related outcomes.

Then, Calculator application with seed 2 was tested using ET. No test cases were provided to the subjects. Therefore, they had to perform free testing and they were not bound to follow any test cases or test steps. They only needed to $\log$ the identified bugs in the bug sheet. Bug report template was also given to the subjects in order to log the identified bugs.

Finally, Calculator application with seed 3 was tested using HT. The RBTCs and test missions were provided to the subjects. They executed the test missions after completely performing the RBTC. While performing a test mission, subjects were told that they should look into the specified area as mentioned on 
the test sheet but they can use their ideas and follow whatever steps they want to. At the same time they should also write down some high-level test steps, which they performed during a session. Upon completion, all relevant material including the test cases, RBTCs, test sessions and bug sheets were collected.

Validity Threats: For the experimental study the following validity threats have to be considered. The application, which was used for the experiment was a simple calculator with eleven functionalities. We do not know how the results would have been influenced if this had been a larger and more complex application. Therefore, the conclusions of this experiment are limited, but were useful for formulating propositions for future studies. However, as pointed out earlier the application reduced the threat of learning effect as how a calculator works is common knowledge. Hence, there was a trade-off to be made between a complex application and learning effect.

On the other hand, even though we controlled the learning effect with respect to the application to some extent, there still exist a learning effect for how to test the application. In order to evaluate an as realistic scenario as possible from an industrial standpoint (a company developing a software using ST adopts ET and later on evolves it to HT with the software having changes in defect population over time) the learning effect could only partially be controlled. That is, in the first run testers had test cases given to the subjects, and with that had ideas what inputs they could use. Hence, they could utilize this knowledge during their testing in the next step (free exploration). Furthermore, if they are finding new interesting tests, this knowledge could again be used to improve. Overall, this is a threat to control the learning effect, while in practice the situation would be similar, where when adopting a new practice, the testers would carry on the knowledge to the new practice. Hence, this is a threat to theoretical validity, while it strengthens external validity.

Another validity threat is the limitation of the FC measure used in relation to extent of testing. As many functions have more than one defect, it is possible that the function was marked as covered by identifying only one defect and other defects in that function might have remained undetected. Such scenario is possible but we think that this should not cause too much impact on the experiment outcomes as the seeded defects were of similar types and related to functionality of a function. Therefore, there is a limited chance that one might find a defect related to functionality and missed the other similar defect related to same functionality.

Another threat to validity is the sample size used. Three treatments, each having six subjects were tested. The small number of subjects in each group could be a potential threat from two perspectives: a practical perspective and a statistical perspective. In order to increase the statistical power, we first reduced the subject heterogeneity by having a background check and using subjects from only one company. Furthermore, we used a powerful statistical test. Our power calculations showed both large effect size and power for the significant differences observed. 
However, we should note that a threat from a practical perspective still exists as, if the subjects were not completely homogenous, the effect of the difference between them could not be ruled out given that the sample is not representative for a larger population of testers. Furthermore, no novice testers were part of the experiment. That is, more data points would reduce the risk of lack of heterogeneity.

Finally, the experimental study was scoped to testing quality (defect detection effectiveness and functionality coverage). However, cost effectiveness, customer satisfaction and some other aspects of the nature of processes (such as risk management etc.) are important outcome variables as well. Hence, they need to be evaluated in further case studies to observe, for example, how much effort is required to test a sufficiently large system in a real world setting, as this would provide rich information on cost or the impacts of HT on customer satisfaction and risk management etc.

Overall, the results hence provide indications that later on can be used as hypotheses in the forthcoming empirical studies (for example, validation studies in the form of controlled experiments or evaluation studies in the form of case studies) in different contexts and further studies. We also would like to emphasize that our analysis provides input to meta-analysis that later allows to aggregate experiments (hence we presented the statistics in detail to allow for that), which with every additional experiment would add an important piece to the overall evidence.

\section{Results and Discussion}

We analyzed the collected data by first evaluating it using descriptive statistics and then performing hypothesis testing.

Defect Detection Effectiveness (DDE): In total, 25 defects were seeded into each version of the application. The numbers of detected defects, found by each subject against each alternative treatment are presented in Table 2 .

Table 2. Defect Detection Effectiveness (The number of detected defects)

\begin{tabular}{cccc}
\hline \multicolumn{5}{c}{ Subject } & ST & ET & HT \\
\hline T1 & 6 & 12 & 10 \\
T2 & 7 & 13 & 11 \\
T3 & 6 & 15 & 12 \\
T4 & 8 & 12 & 10 \\
T5 & 9 & 16 & 13 \\
T6 & 6 & 11 & 8 \\
\hline
\end{tabular}

The descriptive statistics of the three treatments, including median, mean, standard deviation, skewness and kurtosis are given in Table 3 and the box plots for the number of detected defects are shown in Figure 2.

As this experimental study was designed to have 3 treatments to compare (i.e., ET and ST to HT), we first used Analysis of variance (ANOVA), which 
Table 3. Descriptive statistics for the number of detected defects for three treatments

\begin{tabular}{cccccc}
\hline \multicolumn{1}{l}{ Approach } & Median & Mean & Std. Dev. Skewness & Kurtosis \\
\hline ST & 6.5 & 7 & 1.26 & 0.592 & 1.56 \\
ET & 12.5 & 13.16 & 1.94 & 0.425 & 1.43 \\
HT & 10.5 & 10.67 & 1.75 & -0.165 & 1.78
\end{tabular}

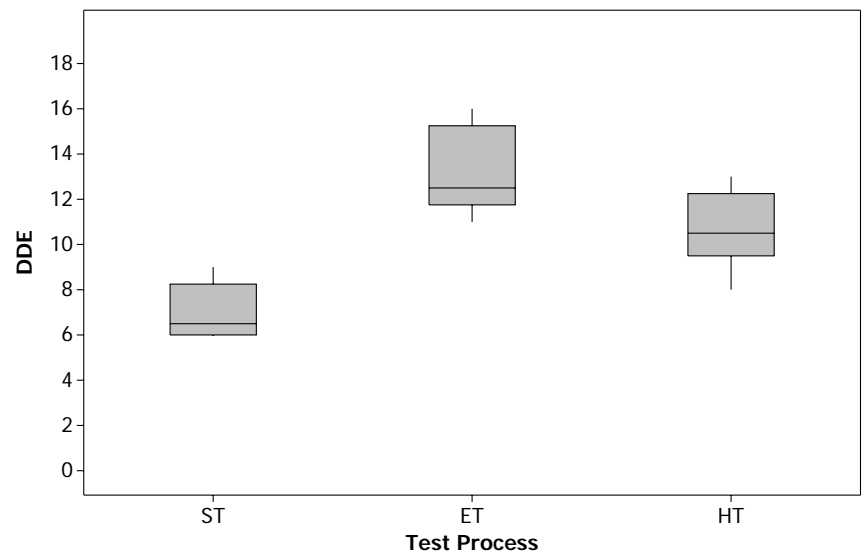

Fig. 2. Box plots of number of detected defects for three treatments

enables detecting significant differences between the treatments as a whole instead of separate t-tests comparing each treatment with another. We chose this analysis technique as it is reliable when being applied to small samples [12].

An important assumption underlying the ANOVA is that all treatments have similar variance. Therefore, we checked the data for "homogeneity of variance". We looked up the table for $F_{\max }[12]$ for the number of treatments in our data and the degrees of freedom (number of replicates per treatment -1 ). The variance ratio, $F$ was found to be 2.2 . As this is smaller than $F_{\max }$ which is 10.8 for 3 treatments and 5 degrees of freedom at $\mathrm{p}$-value $=0.05$, there was no need to transform the data (cf [12]).

Table 4 shows the ANOVA results for DDE, where $u=$ number of treatments and $v=$ number of replicates (The total "Degree of freedom" $(d f)$ is one less than the total no of data entries).

Table 4. ANOVA Summary for DDE

\begin{tabular}{llll}
\hline Source of variance & $\begin{array}{l}\text { Sum } \\
\text { squares (SS) }\end{array}$ & of Degrees of freedom $(d f)$ & $\begin{array}{l}\text { Mean square } \\
(=\mathrm{SS} / d f)\end{array}$ \\
\hline Between groups (bg) & 115.44 & $u-1=2$ & 57.72 \\
Within groups (wg) & $\begin{array}{l}\text { 42.17 } \\
\text { Total }\end{array}$ & $u(v-1)=15$ & 2.81 \\
\hline
\end{tabular}

The tabulated value for the variance ratio for $p=0.05$ was found as $F=3.7$. The variance ratio for the experiment $(F=20.53)$ exceeds this and even exceeds the tabulated $F$ value for $p=0.001(F=11.3)$. This shows that there is a significant difference between treatments and hence $H 1_{0}$ was rejected. 
We further tested the alternative hypotheses by assuming that the calculations of ANOVA are the same as that of a t-test.

First, the least significance difference (LSD) between any of the means is calculated for $p=0.05$ (the level of probability chosen for the $t$ value) using the following formula: $L S D=t-$ value $\times \sigma_{d}$, where $\sigma_{d}^{2}=\frac{2 \times S S / d f}{n}$. Hence, we obtained LSD as 2.92 for t-value $(t=2.13)$ and $\sigma_{d}: 1.37$

Table 5 shows the differences between the means of treatments for DDE as well as LSD $(p=0.05)$. Two means would be significantly different from one another if they differ by more than LSD. The results showed that HT provided higher DDE than ST (i.e., $H 1_{1}$ is accepted) whereas HT provided lower DDE than ET (i.e., $H 1_{2}$ is rejected).

Table 5. The Differences between the Means of Treatments for DDE

\begin{tabular}{lcc}
\hline Treatment pairs & $\overline{\chi_{1}}-\overline{\chi_{2}} L S D=t \times\left(\sigma_{d}\right)$ \\
\hline$D D E_{E T}-D D E_{S T}$ & 6.17 & 2.92 \\
$D D E_{E T}-D D E_{H T}$ & 2.50 & \\
$D D E_{H T}-D D E_{S T}$ & 3.67 & \\
\hline
\end{tabular}

After having performed the ANOVA analysis, we made statistical power analysis [8] ( $t$-test for the difference between two independent means), retrospectively (see Table 6).

Table 6. Power analysis results for the differences between the means of ST, ET and HT treatments for DDE (sample sizes for group 1 and group $2=6, \alpha=0.05, D F=10$, and Critical $t=1.81$ )

\begin{tabular}{lcc}
\hline Pair of treatment Effect size $(\mathrm{d})$ & Power $(1-\beta)$ \\
\hline ET versus ST & 3.77 & 0.99 \\
HT versus ET & 1.35 & 0.70 \\
HT versus ST & 2.41 & 0.99 \\
\hline
\end{tabular}

So, we conclude that our results are significant as both the effect size values and the statistical power are larger than the conventional values [13] (that is Power $=0.80$ and effect size $=0.2$ (small), 0.5 (medium) or 0.80 (large) $)$ despite the small sample size used in this experimental study.

Functionality coverage: The functionality covered by each experiment subjects against each treatment is presented in Table 7.

Table 7. Functionality Coverage (Total number of incorrectly implemented or missing functionalities detected)

\begin{tabular}{|c|c|c|c|}
\hline \multicolumn{4}{|c|}{ Subject ST ET HT } \\
\hline $\mathrm{T} 1$ & 7 & 5 & 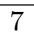 \\
\hline $\mathrm{T} 2$ & 9 & 6 & \\
\hline $\mathrm{T} 3$ & 8 & 9 & 11 \\
\hline $\mathrm{T} 4$ & 7 & 7 & 5 \\
\hline T5 & 8 & 6 & 10 \\
\hline T6 & 8 & 7 & \\
\hline
\end{tabular}

The descriptive statistics of the three treatments, including median, standard deviation, skewness and kurtosis are given in Table 8 and the box plots for the number of detected defects are shown in Figure 3. 
Table 8. Descriptive statistics for functionality coverage of three treatments

\begin{tabular}{|c|c|c|c|c|c|}
\hline Approach & Median & Mean & Std. Dev. & Skewness & Kurtosis \\
\hline $\mathrm{ST}$ & 8 & 7.83 & 0.75 & 0.208 & 1.76 \\
\hline ET & 6.5 & 6.67 & 1,37 & 0.593 & 2.17 \\
\hline $\mathrm{HT}$ & 8.5 & 8.83 & 1.47 & 0.279 & 1.54 \\
\hline
\end{tabular}

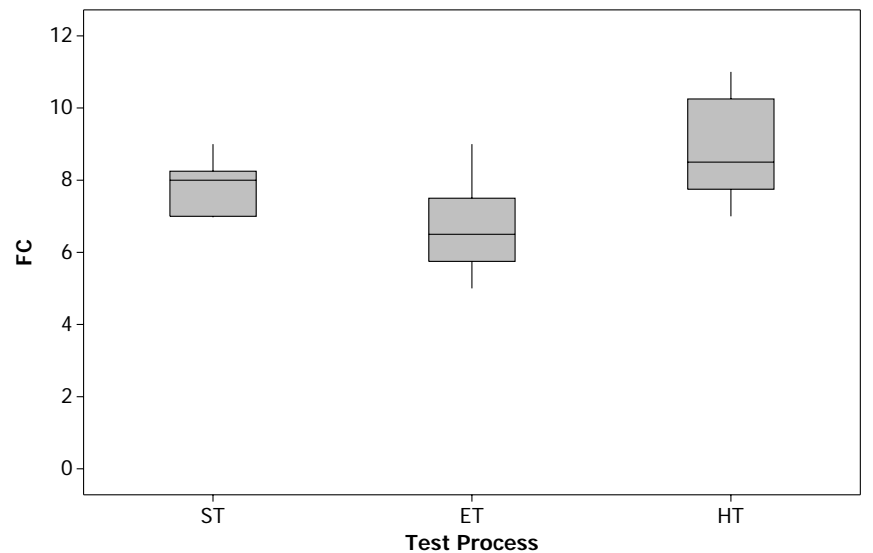

Fig. 3. Box plot of functionality coverage for three treatments

As we did for DDE, we checked the data for FC for "homogeneity of variance". The variance ratio, $F$ was found to be 3.87 . As this is smaller than $F_{\max }$ which is 10.8 for 3 treatments and 5 degrees of freedom at $P=0.05$, there was no need to transform the data.

A single t-test was performed to see if there are differences between the means at a chosen probability level $(p-$ value $=0.05)$. Table 9 shows the values of sum of squares for FC.

Table 9. ANOVA Summary for FC

\begin{tabular}{llll}
\hline Source of variance & $\begin{array}{l}\text { Sum } \\
\text { squares (SS) }\end{array}$ & of Degrees of freedom $(d f)$ & $\begin{array}{l}\text { Mean square } \\
(=\mathrm{SS} / d f)\end{array}$ \\
\hline Between groups (bg) & 14.11 & $u-1=2$ & 7.06 \\
Within groups (wg) & 23 & $u(v-1)=15$ & 1.53 \\
Total & 37.11 & $(u v)-1=17$ & \\
\hline
\end{tabular}

The tabulated value of $F(p=0.05)$ was found as 3.7. Our calculated $F$ value $(F=4.6)$ is higher than this limit, meaning that there is a significant difference between the treatments. Therefore, the null hypotheses $H 2_{0}$ was rejected.

We further tested the alternative hypotheses by calculated the least significance difference (LSD) between the two means and comparing this value to the differences between the means of two treatments. Table 10 shows the differences between the means of treatments for FC as well as LSD $(p=0.05)$.

Table 10. The Differences between the Means of Treatments for FC

\begin{tabular}{lcc}
\hline Treatment pairs $\overline{\chi_{1}}-\overline{\chi_{2}}$ & $L S D=t \times\left(\sigma_{d}\right)$ \\
\hline$F C_{E T}-F C_{S T}$ & 1.16 & 2.15 \\
$F C_{H T}-F C_{S T}$ & 1 & \\
$F C_{H T}-F C_{E T}$ & 2.16 & \\
\hline
\end{tabular}


The results showed that only the difference between the means of HT and ET (at $p=0.05$ ) is slightly greater than 2.15 . Therefore, we conclude that HT provided higher FC than ET (i.e., $\mathrm{H}_{2}$ is accepted)). No significant differences were observed between the means of $\mathrm{HT}$ and ST (i.e., $H 2_{1}$ is rejected).

After having performed the ANOVA analysis, we again made a post hoc (retrospective) statistical power analysis. The results are shown in Table 11.

So, we conclude that our result for the difference between HT and ET is significant as both the effect size value and the statistical power are larger than the conventional values [13]. As for the differences between HT and ST as well as ST and ET, no significance was observed between the means, which might be due to low statistical power values associated with the tests and, therefore should be further investigated.

Table 11. Power analysis results for the differences between the means of ST, ET and HT treatments for FC (sample sizes for group 1 and group $2=6, \alpha=0.05, D F=10$, and Critical $t=1.81$ )

\begin{tabular}{lcc}
\hline Pair of treatment Effect size $(\mathrm{d})$ & Power $(1-\beta)$ \\
\hline ET versus ST & 1.05 & 0.52 \\
HT versus ET & 1.52 & 0.80 \\
HT versus ST & 0.86 & 0.40 \\
\hline
\end{tabular}

As a summary, our alternative hypothesis tests showed that in terms of defect detection effectiveness, HT performed better than ST and worse than ET. In terms of functionality coverage, HT performed better than ET.

Experience: When different subjects are compared with respect to their performance considering their experience levels:

- All subjects were more successful in terms of DDE when they implemented ET. Testers T3 and T5 who hold the maximum level of testing experience (more than ten years) performed better than other subjects when they used either ET or HT. Interestingly, tester T3 was not very effective in terms of DDE when he used ST and in terms of FC when he used ET. However, both T3 and T5 outperformed in terms of FC when they implemented HT.

- Testers T1, T2 and T4, who hold same level of experience (five years), were also similar in detecting defects when they used the same approach. They all found higher number of defects implementing ET as compared to HT and ST. For FC, these subjects on the average covered the same number of functionalities implementing the ST and HT. However, they were not as successful in terms of FC when they implemented ET.

- Tester T6 who holds the less experience among all, detected more defects in comparison to ST when implemented ET and HT, but still less than the other subjects. For FC on the other hand, this subject performed similarly as the other moderate level experienced subjects.

From the box-plots in combination with the observations mentioned above some interesting propositions can be derived that should be checked in larger, controlled experiments and industrial case studies. Note that these observations 
were made in a situation where limited amount of time is available for testing, and that no novice testers participated in the experiment.

- FC: Figure 5 and Table 17 show descriptive statistics for all three treatments. The median values for ST and HT are very close whereas HT shows much higher standard deviation. Highly experienced testers (T3 and T5 with more than 10 years of experience) perform best in terms of FC when using HT. With a moderate level of experience (3-5 years) ST and HT seem to perform similar. When comparing ET and HT we could see that each tester achieves higher test coverage with HT than ET (see Table 7).

- DDE: Figure 4 and Table 12 show that ET outperforms ST and HT. For each of the testers we could see that they perform better with ET and HT than with ST. Furthermore, every tester performed better with ET than with HT. The standard deviation shows that experienced testers benefit more from having freedom in exploring.

\section{Conclusion}

In this research we conducted a experimental study comparing a hybrid testing process incorporating scripted and exploratory testing. The study was conducted with industry practitioners.

The results indicate that $\mathrm{HT}$ is more effective in defect detection than ST and less effective than ET. In terms of functionality coverage, HT performs better than ET. As the statistical power results showed both large effect size and power, we conclude that our results are significant. On the other hand, for FC, no significant differences between the means of HT and ST as well as ST and ET were observed. This might also be due to low statistical power which should be tested in future studies. These results indicate that the HT process provides a compromise solution for testing quality.

Implications related to experience: Looking at the individual data points in the results, some interesting patterns were also brought into light, which led to the following proposition: If one aims at high DDE then ET process should be chosen, especially for experienced testers. However, if FC is important at the same time, HT performed best for experienced testers, but not worse with less experienced testers. Hence the optimum solution for high DDE and FC would be to choose HT. Only using ST is not recommended as after the experiment testers reported that they did not like to use ST as it limits their creativity and does not allow them to bring in their experience and competence, which was also reflected in the poor results ST achieved in comparison to ET and HT with respect to DDE. Given the limitations of the experimental study (see validity threats), these statements only provide indications that need to be further evaluated in future studies.

Furthermore, which process to choose seems to depend also on the experience level (or may be skills) of the tester. Our final observations revealed HT to perform best for experienced testers, but worse with less experienced testers. 
Therefore, the skills, experience and domain knowledge of testers should also be taken into account for future studies. For example, Itkonen et al. [16] and Juristo et al. [20] found that different testers have varying test case designs even though they follow the same test case design strategy. That is, there are indications that the outcome of the test case design varies with individual experience and skill. A survey of literature related to comparisons between novices and experts has shown that experience has positive effects on various parameters. For example, Sim et al. [25] reported that experience has a positive effect with respect to domain knowledge, speed of completing tasks, ability to identify meaningful patterns, superior recall because experts organize short-term and long-term memory more efficiently, etc.

Our study contributes to highlight the importance of experience. In future work experiments should focus on exploring the role of experience further.

HT and agile: It was observed that with time-boxed iterations there is limited time for testing, in particular fitting enough testing in an iteration is perceived a challenge [22]. Hence, even though not widely considered an agile practice, the flexibility that HT provides (in particular for experienced testers) merits further investigations to understand how to integrate HT as an agile practice into the set of agile practices, and how it can be beneficial when combined with other agile practices (such as time-boxing).

\section{References}

1. ISO/IEC: 29119-2: Test processes, international software testing standard. Technical report (2013)

2. Agruss, C., Johnson, B.: Ad hoc software testing, a perspective on exploration and improvisation. Technical report, Florida Institute of Technology, USA (April 2000)

3. Itkonen, J., Mäntylä, M., Lassenius, C.: How do testers do it? an exploratory study on manual testing practices. In: Proceedings of the Third International Symposium on Empirical Software Engineering and Measurement (ESEM 2009). (2009) 494-497

4. Ahonen, J.J., Junttila, T., Sakkinen, M.: Impacts of the organizational model on testing: Three industrial cases. Empirical Software Engineering 9(4) (2004) $275-296$

5. Andersson, C., Runeson, P.: Verification and validation in industry - a qualitative survey on the state of practice. In: International Symposium on Empirical Software Engineering (ISESE 2002). (2002) 37-47

6. Itkonen, J., Rautiainen, K.: Exploratory testing: a multiple case study. In: International Symposium on Empirical Software Engineering (ISESE 2005). (2005) $84-93$

7. Itkonen, J.: Do test cases really matter? An experiment comparing test case based and exploratory testing. PhD thesis, Helsinki University of Technology, Finland (2008)

8. Kaner, C., Falk, J., Nguyen, H.Q.: Testing computer software. 2. ed. edn. Van Nostrand Reinhold, New York (1993) 
9. Crispin, L., Gregory, J.: Agile Testing: A Practical Guide for Testers and Agile Teams. Addison-Wesley (2009)

10. Itkonen, J., Rautiainen, K., Lassenius, C.: Toward an understanding of quality assurance in agile software development. International Journal of Agile Manufacturing 8(2) (2005) 39-49

11. Bourque, P., Dupuis, R.: Guide to the software engineering body of knowledge (swebok). Technical report, IEEE Computer Society, Los Alamitos, California (2004)

12. Bach, J.: Exploratory testing. In Veenendal, E.V., ed.: The Testing Practitioner. UTN Publishers (2005)

13. Bach, J.: Session-based test management. Software Testing and Quality Engineering Magazine 2 (2000)

14. Shoaib, L., Nadeem, A., Akbar, A.: An empirical evaluation of the influence of human personality on exploratory software testing. In: Proceedings of the IEEE 13th International Multitopic Conference (INMIC 2009). (2009) 1-6

15. Copeland, L.: A practitioner's guide to software test design. Artech House, Boston, Mass. (2004)

16. Itkonen, J., Mäntylä, M., Lassenius, C.: Defect detection efficiency: Test case based vs. exploratory testing. In: Proceedings of the First International Symposium on Empirical Software Engineering and Measurement (ESEM 2007). (2007) 61-70

17. Shah, S.M.A., Gencel, C., Alvi, U.S., Petersen, K.: Towards a hybrid testing process unifying exploratory testing and scripted testing. Journal of Software Maintenance and Evolution: Research and Practice (2013)

18. Tahat, L.H., Bader, A., Vaysburg, B., Korel, B.: Requirement-based automated black-box test generation. In: Proceedings of the 25th International Computer Software and Applications Conference (COMPSAC 2001). (2001) 489-495

19. Basili, V.R., Caldiera, G., Rombach, H.D.: The goal question metric approach. Encyclopedia of software engineering 2(1994) (1994) 528-532

20. Wohlin, C.: Experimentation in software engineering: an introduction. Kluwer, Boston (2000)

21. Dybå, T., Kampenes, V.B., Sjøberg, D.I.K.: A systematic review of statistical power in software engineering experiments. Information \& Software Technology 48(8) (2006) 745-755

22. David, H.A.: Upper 5 and $1 \%$ points of the maximum f-ratio. Biometrika 39(3) (1952) 422-424

23. Cohen, J.: Statistical power analysis for the behavioral sciences. 2. ed. edn. L. Erlbaum Associates, Hillsdale (1988)

24. Juristo, N., Moreno, A.M., Vegas, S.: Reviewing 25 years of testing technique experiments. Empirical Software Engineering 9(1-2) (2004) 7-44

25. Sim, S.E., Ratanotayanon, S., Aiyelokun, O., Morris, E.: An initial study to develop an empirical test for software engineering expertise. Institute for Software Research, University of California, Irvine, Irvine, CA, USA, Technical Report\# UCI-ISR-06-6 (2006)

26. Petersen, K., Wohlin, C.: A comparison of issues and advantages in agile and incremental development between state of the art and an industrial case. Journal of Systems and Software 82(9) (2009) 1479-1490 